\title{
MÉTODOS CENTRADOS EN EL ESTUDIANTE. SUS EFECTOS EN LAS ESTRATEGIAS Y LOS ENFOQUES DE APRENDIZAJE DE LOS UNIVERSITARIOS
}

\author{
Learning-Student Methods. Their Effects on Learning \\ Strategies and Approaches to Learning of University \\ Students
}

Laura BARBOYON COMBEY y Bernardo GARGALLO LÓPEZ

Universidad de Valencia. España.

laulabar@gmail.com; bernardo.gargallo@uv.es

https://orcid.org/0000-0002-2104-7217

bttps://orcid.org/0000-0002-2805-4129

Fecha de recepción: 23/01/2021

Fecha de aceptación: 01/05/2021

Fecha de publicación en línea: 01/01/2022

Cómo citar este artículo: Barboyon Combey, L., y Gargallo López, B. (2022). Métodos centrados en el estudiante. Sus efectos en las estrategias y los enfoques de aprendizaje de los universitarios. Teoría de la Educación. Revista Interuniversitaria, 34(1), 215-237. https://doi.org/10.14201/teri.25600

\section{RESUMEN}

En la universidad nos encontramos en un proceso de cambio, desde un modelo centrado en la enseñanza a otro centrado en el aprendizaje o en el alumno. Es relevante disponer de datos científicos de aplicación de este tipo de metodologías para evaluar tanto su utilidad como su funcionalidad. El objetivo de esta investigación fue valorar si la aplicación de métodos centrados en el aprendizaje provocaría mejora en las estrategias y los enfoques de aprendizaje de los alumnos con respecto al grupo control, el cual recibió formación con un formato metodológico centrado en la 
enseñanza. Se hizo uso de un diseño cuasiexperimental, de cohortes, con grupo de control no equivalente. La muestra estaba integrada por 243 estudiantes de Pedagogía y Educación Social de la Universidad de Valencia, que cursaban una materia de $1^{\circ}$ curso. Los métodos utilizados buscaban el compromiso y la participación activa del alumnado haciendo uso de preguntas, simulaciones, trabajo cooperativo, actividades prácticas, realización de un trabajo de investigación, portafolios, etc. Los resultados que se obtuvieron fueron positivos. Los estudiantes que recibieron intervención educativa con métodos centrados en el alumno mejoraron sus estrategias de aprendizaje e incrementaron el enfoque profundo de aprendizaje en comparación con sus compañeros que habían cursado la misma asignatura con métodos tradicionales. Estos resultados son un exponente de la validez de estos métodos y pueden servir de motivación al profesorado para que se decante por un enfoque centrado en el aprendizaje, habida cuenta, además, de que su aplicación es relativamente fácil de articular.

Palabras clave: estudiantes universitarios; enseñanza centrada en el aprendizaje; estrategias de aprendizaje; enfoques de aprendizaje; métodos centrados en la enseñanza.

\section{ABSTRACT}

At the university we are in a process of change, from a teaching centered approach to a learning centered approach. It is important to have scientific data about the use of such methodologies to evaluate both its usefulness and its functionality. The aim of this research was to assess whether the application of learning-centered methods would lead to an improvement in students' learning strategies and approaches to learning compared to the control group, which received training with a teaching-centered format. A quasi-experimental cohort design, with a non-equivalent control group. The sample consisted of 243 Pedagogy and Social Education students at the University of Valencia, who were studying a course in their 1st year. The methods we used were seeking the commitment and active participation of students, by using questions, simulations, cooperative work, practical activities, development of a research project, portfolios, etc. The results that we get were positive. The students who received educational intervention with the leaning centered approach improved their learning strategies and increased the deep approach of learning compared to their peers who had studied the same course with traditional methods. These results prove the validity of these methods and can serve to motivate teachers to choose a learning centered approach; moreover, because it is relatively easy to apply these methods in the classroom.

Key words: university students; learning centered teaching; learning strategies; learning approaches, teaching centered approach.

\section{INTRODUCCIÓN}

En la historia de la educación se encuentran aproximaciones en que el aprendiz es el centro del proceso de formación. Hoy hablaríamos de enfoques centrados 
en el estudiante o en el aprendizaje. Algunos hitos significativos son la orientación rousseauniana o iniciativas como las de Montessori, Decroly, Claparède, Cousinet o Freinet, en la línea de la Escuela Nueva o de la Educación Progresista, entre otras. En España es especialmente significativa la Institución Libre de Enseñanza, de raíz krausista. También se encuentran aportaciones de figuras relevantes como Piaget o Vygotsky, que cuestionaron el conductismo, dando forma a nuevas teorías más relevantes que explicaban mejor el proceso de aprendizaje, al tiempo que preconizaban la autonomía del alumno, la necesidad de una construcción personal de significados y la colaboración con los otros.

A finales del siglo pasado se producen cambios en los modelos de enseñanzaaprendizaje en la universidad, que condujeron a nuevos planteamientos. Barr y Tagg (1995), en un trabajo inicial de referencia, planteaban la necesidad de un cambio de paradigma en la formación en la Educación Superior.

Barr y Tagg (1995) defendían que la universidad ha funcionado con un enfoque de formación centrado en la instrucción y en el profesor, el "paradigma instruccional", - hoy diríamos enfoque centrado en la enseñanza/en el profesor (teacher-centered learning) — , basado en un modelo de profesor que "sabe» y que "distribuye» el conocimiento a sus alumnos con metodología expositiva. En este paradigma se entiende que la clave del proceso de enseñanza-aprendizaje es una buena instrucción.

El proceso formativo se interpreta como algo acumulativo, de manera que el "todo» es la suma de las partes, de las asignaturas y de los créditos conseguidos. Las materias y los departamentos que las imparten tienen vida propia y se «defienden» frente a la agresión externa. Los profesores defienden su "territorio» frente a los "Otros", luchando para que su asignatura esté presente en los programas y cuanta más presencia tenga, mejor, teniendo en poca consideración las competencias o necesidades de los estudiantes, cuando se diseñan planes de estudio.

Por el contrario, el paradigma centrado en el aprendizaje (student-centered learning; learning paradigm) sitúa al alumno en el centro del proceso de formación, buscando crear entornos de aprendizaje que le permitan construir conocimiento y trabajar con los otros para resolver problemas complejos.

Dar el cambio a este paradigma demanda acabar con la estructura compartamentalizada del paradigma instruccional y diseñar el currículum y los títulos académicos identificando las competencias que deben lograr los graduados. A partir de ahí se concretan los resultados de aprendizaje que ilustran su logro, y se seleccionan los contenidos, métodos y procesos formativos. En este planteamiento, que conecta plenamente con el alineamiento constructivo (Biggs, 2005), el todo es más que la suma de las partes y es lo que organiza y gobierna las partes. La idea no es que el estudiante acumule créditos, sino que demuestre que ha logrado los aprendizajes necesarios, concretados en resultados de aprendizaje relevantes. 
LAURA BARBOYON COMBEY Y BERNARDO GARGALLO LÓPEZ

MÉTODOS CENTRADOS EN EL ESTUDIANTE. SUS EFECTOS EN LAS ESTRATEGIAS

Y LOS ENFOQUES DE APRENDIZAJE DE LOS UNIVERSITARIOS

Trabajar en esta línea exige que la evaluación sea de calidad y que permita valorar el logro de esos resultados que demuestren el dominio de las competencias.

Aparte del trabajo de Barr y Tagg (1995), teórico, hay trabajos empíricos, llevados a cabo con profesorado de la universidad, que lo complementan analizando el proceso de enseñanza-aprendizaje a partir de la percepción de los profesores y concretando cómo entienden la enseñanza, el aprendizaje, la evaluación y la construcción del conocimiento.

Para estas investigaciones se ha utilizado metodología cualitativa, analizando las creencias de los profesores sobre estos tópicos y también lo que dicen que hacen cuando se ocupan de enseñar. El enfoque adoptado es fenomenográfico/ interpretativo y se ha hecho uso de la entrevista. Como ejemplo de estos trabajos tenemos los de Gow y Kember (1993), Kember y Gow (1994) y Samuelowicz y Bain (1992, 2001), entre otros.

Partiendo de estos trabajos se puede confirmar la coexistencia de un modelo tradicional, centrado en la enseñanza/en el profesor, y de un modelo centrado en el aprendizaje/en el alumno/constructivista ${ }^{1}$, que ha ido incrementando su influencia en el proceso de formación (McCoy et al., 2018). Ambos se diferencian tanto en la enseñanza como en la evaluación.

Otras investigaciones, algunas muy recientes, corroboran la pervivencia de ambos modelos (Arman, 2018; Biggs, 2005; EI-ESU, 2010; Emaliana, 2017; García Valcárcel, 1993; Kember, 2009; Kember y Kwan, 2000; Motjolopane, 2021). El hecho de que el modelo centrado en el aprendizaje esté asentado a nivel teórico desde finales del siglo XX no significa que sea el predominante a nivel pragmático. Todavía hoy la lección magistral es el método más generalizado entre el profesorado universitario (Jiménez Hernández, González Ortiz y Tornel Abellán, 2020), es verdad que bastantes veces complementado con otros métodos.

Las características más significativas de los dos modelos son las que siguen:

El modelo centrado en la enseñanza da más relevancia al profesor. Su principal función es transmitir sus conocimientos y la de los alumnos reproducirlos (Kember, 2009; Samuelowicz y Bain, 2001). El método más utilizado es la lección magistral. El profesor explica, el alumno toma apuntes, memoriza y reproduce esa información en un examen, el instrumento más frecuente utilizado para evaluar.

En cambio, el modelo centrado en el aprendizaje enfatiza el aprendizaje del alumnado. Se inscribe en la perspectiva constructivista, entendiendo el conocimiento como una construcción personal del alumno. El objetivo es que los estudiantes

1. En las primeras publicaciones aje no se asociaba explícitamente el modelo centrado en el aprendizaje con la concepción constructivista; sin embargo, hoy hay acuerdo en que este modelo es constructivista (EI-ESU, 2010; Carwile, 2007; Lavoie y Rosman, 2007; Serin, 2018), al enfatizar la construcción del conocimiento por parte de los estudiantes, la importancia del aprendizaje cooperativo, el papel del profesor como mediador, el uso de metodologías activas y la relevancia de la interactividad, el uso de evaluación formativa, etc. 
desarrollen las habilidades, actitudes y valores que necesitarán a lo largo de toda su vida. Se usan métodos que fomentan la implicación del alumnado, complementarios de la lección magistral (trabajo cooperativo, proyectos, aprendizaje basado en problemas, estudio de casos, contrato de aprendizaje, etc.) y la evaluación es continua/formativa. El objetivo del profesor en este modelo es que los alumnos aprendan a aprender.

El proceso de cambio hacia este modelo se ha visto potenciado con el desarrollo del Espacio Europeo de Educación Superior, ya que el modelo de Bolonia se decanta por una universidad centrada en el aprender (CE, 2007, 2009, 2012, 2015).

Disponer de datos de intervenciones fructíferas puede ayudar al profesorado a decantarse por esta opción. Es lo que pretendemos analizar en este trabajo, valorando el impacto de métodos centrados en el alumno sobre el aprendizaje de los universitarios.

Por ello presentamos resultados derivados una investigación que comportaba la implementación de métodos centrados en el aprendizaje, por profesorado de la Universidad de Valencia.

Para analizar su impacto en el aprendizaje del alumnado se evaluaron las estrategias de aprendizaje de los estudiantes, que permiten concretar cómo despliegan las habilidades, técnicas y procedimientos para aprender eficazmente, y también su capacidad de autorregulación metacognitiva (Pintrich, 2000 y 2004; Yip, 2012; Zimmerman, 2002). Se evaluaron, también, los enfoques de aprendizaje, que permiten concretar en qué medida un estudiante afronta el aprendizaje con un enfoque profundo, o con un enfoque superficial (Entwistle y Peteron, 2004; McCune y Entwistle, 2011; Ruiz et al., 2008).

\section{MÉTOdo}

\subsection{Objetivos}

Objetivo 1. Evaluar el impacto de un formato metodológico centrado en el aprendizaje, aplicado por tres profesores diferentes de dos titulaciones universitarias, en el modo de aprender del alumnado, concretado en sus estrategias y enfoques de aprendizaje.

Objetivo 2. Analizar la influencia del profesor en la eficacia de dicho formato.

\subsection{Diseño y fases de la investigación}

Se utilizó un diseño cuasiexperimental no equivalente pretest-postest, con grupo experimental y de control (Campbell y Stanley, 1979; Shadish et al., 2002).

La variable clave es el formato de docencia y evaluación de los profesores, centrado en el aprendizaje, para determinar su influencia en las variables evaluadas en los estudiantes. Dentro de los diseños cuasiexperimentales se ha optado por un 
diseño de cohortes, de grupos naturales de clase, que comparten características vitales y que reciben intervención específica (Ato y Vallejo, 2007; Shadish et al., 2002).

En ese caso se toman como cohortes grupos sucesivos que realizan el mismo curso $\left(1^{\circ}\right)$, en una misma materia de diferente titulación y año académico, de modo que los grupos del primer curso académico (2016-17) (agrupados en el gráfico bajo la denominación Grupo 1) no reciben la intervención específica que sí sufren los del segundo curso académico (2017-18) (agrupados bajo la denominación Grupo 2). Por lo tanto, la cohorte del primer año será utilizada como grupo de control (Shadish et al., 2002).

Llevamos a cabo una primera toma de datos (postest, O1) sobre los grupos de control en el primer año de la investigación, finalizadas sus clases con metodología tradicional. Al año siguiente se implantó la metodología centrada en el aprendizaje y se realizaron el pretest (O2) y el postest (O3) en los grupos experimentales. Este diseño posibilita la comparación de los grupos experimentales — con intervencióny los de control, y el análisis de la evolución de los grupos experimentales, del segundo año, del pretest al postest.

FIGURA 1

GRÁFICO DEL DISEÑO DE LA INTERVENCIÓN

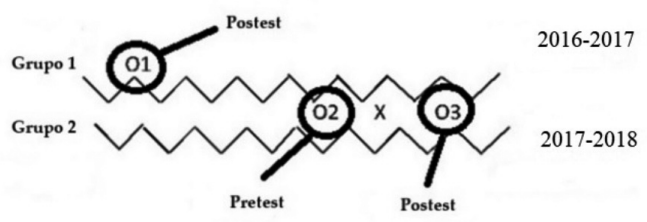

Fuente: Elaboración propia

\subsection{Población y muestra}

La muestra está integrada por 243 estudiantes, 64 del primer curso y 179 del segundo, de Pedagogía y Educación Social de la Universidad de Valencia, cursando la misma materia de $1^{\circ}$ curso.

El Grupo 1 se compone de dos subgrupos en que dos profesores, A y B, imparten la misma materia. El profesor A lo hace en el grupo de Educación Social (34 alumnos) y el profesor B en el de Pedagogía (30 alumnos). El Grupo 2 está integrado por tres subgrupos impartidos por tres profesores, A, B y C, impartiendo la misma materia que el año anterior. Los profesores A y B lo hacen en dos grupos de Pedagogía (75 y 53 alumnos respectivamente) y el profesor C, que no intervino el curso anterior, en el grupo de Educación Social (51 alumnos). 


\subsection{Instrumentos}

Para evaluar las estrategias de aprendizaje se utilizó el cuestionario CEVEAPEU de Gargallo et al. (2009), de 88 ítems, dos escalas, seis subescalas y veinticinco estrategias (Tabla 1). La fiabilidad-consistencia interna del cuestionario es de $\alpha=.897$ y la de las dos escalas $\alpha=.819$ y $\alpha=.864$. Tiene un buen índice de consistenciaestabilidad temporal y un buen valor predictivo sobre el rendimiento.

\begin{tabular}{|c|c|c|}
\hline Escalas & Subescalas & Estrategias \\
\hline \multirow{15}{*}{$\begin{array}{l}\text { Estrategias } \\
\text { afectivas, } \\
\text { de apoyo } \\
\text { y control/ } \\
\text { automanejo }\end{array}$} & \multirow{7}{*}{$\begin{array}{l}\text { Estrategias motivacionales } \\
\qquad(\alpha=, 692)\end{array}$} & Motivación intrínseca $(\alpha=, 500)$ \\
\hline & & Motivación extrínseca $(\alpha=, 540)$ \\
\hline & & Valor de la tarea $(\alpha=, 692)$ \\
\hline & & Atribuciones internas $(\alpha=, 537)$ \\
\hline & & Atribuciones externas $(\alpha=, 539)$ \\
\hline & & Autoeficacia y expectativas ( $\alpha=, 743$ ) \\
\hline & & $\begin{array}{c}\text { Concepción de la inteligencia como } \\
\text { modificable }(\alpha=595)\end{array}$ \\
\hline & \multirow{2}{*}{$\begin{array}{l}\text { Componentes afectivos } \\
\qquad(\alpha=, 707)\end{array}$} & Estado físico y anímico $(\alpha=, 735)$ \\
\hline & & Ansiedad $(\alpha=, 714)$ \\
\hline & \multirow{4}{*}{$\begin{array}{l}\text { Estrategias metacognitivas } \\
\qquad(\alpha=, 738)\end{array}$} & $\begin{array}{c}\text { Conocimiento de objetivos y criterios de } \\
\text { evaluación }(\alpha=, 606)\end{array}$ \\
\hline & & Planificación $(\alpha=, 738)$ \\
\hline & & Autoevaluación $(\alpha=, 521)$ \\
\hline & & Control, autorregulación $(\alpha=, 660)$ \\
\hline & \multirow{2}{*}{$\begin{array}{l}\text { Estrategias de control del } \\
\text { contexto, interacción social } \\
\text { y manejo de recursos } \\
(\alpha=, 703)\end{array}$} & Control del contexto $(\alpha=, 751)$ \\
\hline & & $\begin{array}{c}\text { Habilidades de interacción social y } \\
\text { aprendizaje con compañeros } \\
(\alpha=, 712)\end{array}$ \\
\hline
\end{tabular}


LAURA BARBOYON COMBEY Y BERNARDO GARGALLO LÓPEZ MÉTODOS CENTRADOS EN EL ESTUDIANTE. SUS EFECTOS EN LAS ESTRATEGIAS Y LOS ENFOQUES DE APRENDIZAJE DE LOS UNIVERSITARIOS

\begin{tabular}{|c|c|c|}
\hline Escalas & Subescalas & Estrategias \\
\hline \multirow{10}{*}{$\begin{array}{l}\text { Estrategias } \\
\text { relacionadas } \\
\text { con el } \\
\text { procesamiento } \\
\text { de la } \\
\text { información } \\
\text { (a=,864) }\end{array}$} & \multirow{5}{*}{$\begin{array}{l}\text { Estrategias de búsqueda y } \\
\text { selección e información } \\
\qquad(\alpha=, 705)\end{array}$} & $\begin{array}{c}\text { Conocimiento de fuentes y búsqueda de } \\
\text { información }(\alpha=, 685)\end{array}$ \\
\hline & & Selección de información $(\alpha=, 630)$ \\
\hline & & Adquisición de información $(\alpha=, 677)$ \\
\hline & & Elaboración $(\alpha=, 739)$ \\
\hline & & Organización $(\alpha=, 810)$ \\
\hline & \multirow{5}{*}{$\begin{array}{l}\text { Estrategias de } \\
\text { procesamiento y uso de la } \\
\text { información } \\
(\alpha=, 821)\end{array}$} & $\begin{array}{l}\text { Personalización y creatividad, } \\
\text { pensamiento crítico }(\alpha=, 771)\end{array}$ \\
\hline & & $\begin{array}{l}\text { Almacenamiento, Memorización, Uso de } \\
\text { recursos mnemotécnicos }(\alpha=, 765)\end{array}$ \\
\hline & & $\begin{array}{l}\text { Almacenamiento, Simple repetición } \\
(\alpha=, 691)\end{array}$ \\
\hline & & $\begin{array}{l}\text { Transferencia, Uso de la información } \\
\qquad(\alpha=, 656)\end{array}$ \\
\hline & & $\begin{array}{l}\text { Manejo de recursos para usar la } \\
\text { información adquirida }(\alpha=, 598)\end{array}$ \\
\hline
\end{tabular}

Fuente: Gargallo, Garfella, Sahuquillo, Verde, y Jiménez (2015).

Para evaluar los enfoques de aprendizaje se utilizó el cuestionario CPE, traducción española del R-SPQ-2F de Biggs et al. (2001), de 20 ítems divididos en dos escalas: enfoque superficial (10 ítems) y enfoque profundo (10 ítems), subdivididos en dos factores: motivos (superficial y profundo) y estrategias (superficial y profunda) (Gargallo, Garfella, Sahuquillo, Verde, y Jiménez, 2015). La Tabla 2 presenta su estructura y datos de fiabilidad.

TABLA 2

ESTRUCTURA Y DATOS DE FIABILIDAD-CONSISTENCIA INTERNA DEL CUESTIONARIO CPE

Escalas

\begin{tabular}{ll}
\hline \multicolumn{1}{c}{ Escalas } & \multicolumn{1}{c}{ Factores } \\
\hline I. Enfoque profundo $(\alpha=, 812)$ & Motivo profundo $(\alpha=, 631)$ \\
\cline { 2 - 2 } II. Enfoque superficial $(\alpha=, 795)$ & Motrategia profunda $(\alpha=, 688)$ \\
\cline { 2 - 2 } & Estrategia superficial $(\alpha=, 706)$ \\
\hline
\end{tabular}

Fuente: Gargallo, Garfella, Sahuquillo, Verde, y Jiménez (2015). 
LAURA BARBOYON COMBEY Y BERNARDO GARGALLO LÓPEZ

MÉTODOS CENTRADOS EN EL ESTUDIANTE. SUS EFECTOS EN LAS ESTRATEGIAS

Y LOS ENFOQUES DE APRENDIZAJE DE LOS UNIVERSITARIOS

\subsection{Procedimiento}

El alumnado del grupo 1 respondió los cuestionarios al finalizar la docencia de la asignatura en el curso 2016-17, como postest, contextualizando las respuestas en la materia y en la docencia impartida por sus profesores.

El alumnado del grupo 2 los respondió al iniciar la docencia de la materia (pretest) (curso 2017-18), contextualizando sus respuestas en su modo habitual de afrontar el aprendizaje, y los volvió a contestar al terminar la docencia (postest), pensando en la materia en la que estaban y en su profesorado.

El formato metodológico centrado en el aprendizaje utilizado incluye diversos métodos de enseñanza y evaluación y se inscribe en el enfoque de alineamiento constructivo (Biggs, 2005), dándose vinculación entre objetivos, contenidos, tareas, métodos de enseñanza y evaluación.

El profesorado participante utilizaba la misma guía docente y los mismos métodos. Se realizaba una presentación sucinta de las competencias, resultados de aprendizaje, contenidos, tareas y materiales. El alumnado debía trabajar por su cuenta y responder a un repertorio de cuestiones de cada tema por el aula virtual. En clase se debatía sobre las cuestiones elaboradas, de diversos niveles de complejidad, a partir del trabajo autónomo del alumnado. Se llevaba a cabo un primer momento de trabajo en pequeño grupo discutiendo las respuestas y, a continuación, se realizaba una puesta en común en gran grupo, para buscar consensos y aclarar las dudas surgidas, con la ayuda e intervención del profesor, que realizaba las aclaraciones y explicaciones necesarias.

Se realizaban prácticas de los temas para trabajar los conocimientos, habilidades y actitudes programados mediante casos, juegos, simulaciones, vídeos, técnicas de trabajo cooperativo, etc. A lo largo del cuatrimestre realizaban un trabajo cooperativo de investigación sobre el aprendizaje a lo largo de vida en un espacio físico determinado (barrio, ciudad, población pequeña), para analizar los recursos disponibles y su utilización, recogiendo información de informantes clave (autoridades, directores de centros, profesionales, etc.). Debían realizar un informe escrito del trabajo y una presentación oral ante la clase. Esta presentación era evaluada por el profesor y por sus compañeros. Además, cada participante en el trabajo de grupo coevaluaba a sus compañeros, con criterios de evaluación establecidos.

También debían realizar dos entregas de portafolios que recogían evidencias del aprendizaje realizado, incluyendo cuestiones debatidas en clase, informes de las prácticas, informe del trabajo de investigación en grupo, preguntas de reflexión metacognitiva sobre el proceso seguido, autoevaluación y coevaluación del trabajo de grupo. Los profesores ofrecían feedback al alumnado, devolviendo el portafolios corregido, utilizado como instrumento de enseñanza-aprendizaje y de evaluación.

Así pues, los métodos y técnicas de enseñanza utilizados fueron: lección magistral participativa, preguntas sobre la materia, discusión sobre las preguntas, prácticas en el aula —incluyendo diversas técnicas—, elaboración de un trabajo de investigación 
en grupo, trabajo cooperativo, exposiciones y presentaciones del trabajo de grupo y un portafolios. Los métodos y técnicas de evaluación fueron: una prueba escrita de respuesta abierta (40\% de la nota final), un portafolios (60\% de la nota final), la coevaluación y la autoevaluación.

\subsection{Análisis estadísticos}

Se utilizó el programa SPSS 22.0 para Windows realizando ANOVA univariado para la comparación intergrupos. Para la comparación intragrupos se hizo uso de ANOVA mixto (método MLG de medidas repetidas), con estimación del tamaño del efecto ( $\eta 2$ parcial).

\section{RESUltados}

Se presentan organizados en dos bloques: en el primero se toman los sujetos de cada año como un solo grupo, actuando los alumnos de los dos grupos del primer año como grupo de control (metodología tradicional), y los alumnos de los tres grupos de segundo año como grupo experimental (métodos centrados en el aprendizaje). Dentro de este primer bloque, se presentarán primero resultados del análisis intragrupo, de estrategias y enfoques, viendo la evolución del grupo experimental del pretest al postest. A continuación, se presentarán resultados del análisis intergrupos, analizando las diferentes existentes en el postest entre los sujetos experimentales (del segundo año) y los de control (del primer año).

En el segundo bloque se presentarán los resultados obtenidos por los sujetos experimentales (del segundo año) grupo a grupo (análisis intragrupo), ya que, aunque los tres grupos recibieron la misma metodología (centrada en el aprendizaje), pertenecían a tres grupos diferentes de clase, cada uno con un profesor distinto. Esto permitiría analizar el efecto del profesor en el formato centrado en el aprendizaje utilizado.

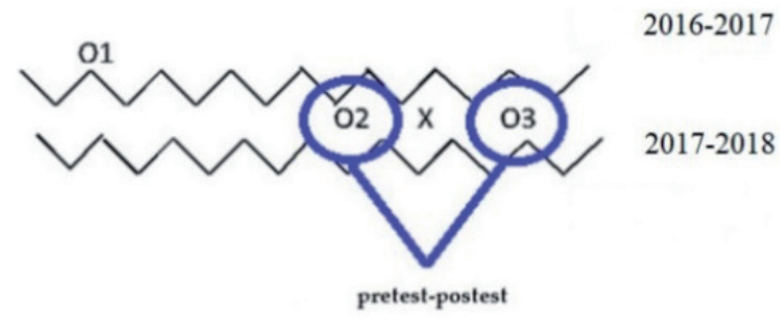

Fuente: Elaboración propia 


\subsection{Resultados obtenidos agrupando a los sujetos de cada año en un solo grupo}

\subsubsection{Análisis intragrupo}

\subsubsection{Resultados en estrategias}

Se encontraron diferencias significativas pretest- postest en la puntuación media global, con mejora en el postest, $\mathrm{p}<.001, \eta^{2}$ parcial $=.152$ (tamaño del efecto grande).

También en la primera escala, de estrategias afectivas, de apoyo y de control/ automanejo, $p<.001, \eta^{2}$ parcial $=.107$ (tamaño del efecto mediano), y en la segunda, de estrategias relacionadas con el procesamiento de la información, $p<$ $.001, \eta^{2}$ parcial $=.142$ (tamaño del efecto grande). Ambas escalas mejoraron las puntuaciones en el postest.

Se dieron diferencias significativas en cinco de las seis subescalas: estrategias metacognitivas, $p<.001, \eta^{2}$ parcial $=.069$, de control del contexto, $p<.001, \eta^{2}$ parcial $=.085$, de búsqueda y selección de la información, $p<.001, \eta^{2}$ parcial $=$ .106 y de procesamiento y uso, $p<.001, \eta^{2}$ parcial $=.114$, todas con tamaño del efecto mediano. En el caso de las estrategias motivacionales, $p<.05, \eta^{2}$ parcial $=$ .033, el tamaño del efecto fue pequeño.

No se encontraron diferencias significativas en la subescala de componentes afectivos, aunque la media fue superior en el postest, y en estado físico/anímico se estuvo cerca del nivel de significación.

Se encontraron diferencias significativas en dieciséis de las veinticinco estrategias:

En tres de las siete estrategias motivacionales: motivación intrínseca, autoeficacia y expectativas $y$ atribuciones externas.

En tres de las cuatro estrategias metacognitivas: conocimiento de objetivos y criterios de evaluación, control y autorregulación/autoevaluación.

Así mismo, en las dos estrategias de control del contexto: control del contexto y habilidades de interacción social.

También en las dos estrategias de búsqueda y selección: conocimiento de fuentes y búsqueda, y selección.

Por fin, en seis de las ocho estrategias de procesamiento y uso: elaboración, organización, personalización y creatividad, pensamiento crítico, almacenamiento, memorización y uso de recursos mnemotécnicos, transferencia y uso de la información, y manejo de recursos para usar la información adquirida.

En las otras estrategias también se dieron mejoras, aunque no estadísticamente significativas.

\subsubsection{Resultados en enfoques}

Se encontraron diferencias significativas pretest-postest en la puntuación media del enfoque superficial, $p<.01, \eta^{2}$ parcial $=.04221$, que descendió, y en la del enfoque profundo, $p<.01, \eta^{2}$ parcial $=.05354$, que creció (tamaño del efecto 
pequeño). También se encontraron diferencias estadísticamente significativas en las puntuaciones parciales, de estrategia y motivo.

\subsubsection{Análisis intergrupos}

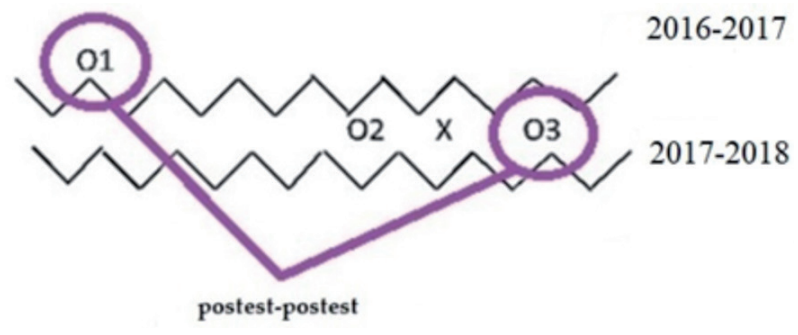

Fuente: Elaboración propia

\subsubsection{Resultados en estrategias de aprendizaje}

Se encontraron diferencias significativas entre ambos postests (2016-2017, 20172018) en la puntuación media global, a favor de los sujetos experimentales, $\mathrm{p}<.001$.

También en la primera escala, de estrategias afectivas, $p<.001$ y en la segunda escala, de estrategias relacionadas con el procesamiento, $p<.001$, a favor de los sujetos experimentales.

Hubo diferencias significativas a favor de los experimentales, en cinco de las seis subescalas: estrategias motivacionales, $p<.001$, metacognitivas, $p<.001$, de control del contexto, $p<.001$, de búsqueda de la información, $p<.01$ y de procesamiento de la información, $p<.001$.

También en dieciocho de las veinticinco estrategias:

En tres estrategias motivacionales: motivación intrínseca, valor de la tarea y autoeficacia y expectativas; en una estrategia afectiva: estado físico y anímico; en las cuatro estrategias metacognitivas: conocimiento de objetivos y criterios de evaluación, planificación, autoevaluación, control y autorregulación; en dos estrategias de control del contexto: control del contexto y habilidades de interacción social; en una estrategia de búsqueda y selección: conocimiento de fuentes y búsqueda de la información; y en siete de las ocho estrategias de procesamiento y uso de la información: adquisición, elaboración, organización, personalización y creatividad, pensamiento crítico, almacenamiento/memorización/uso de recursos mnemotécnicos, almacenamiento por simple repetición y transferencia y uso de la información. 


\subsubsection{Resultados en enfoques de aprendizaje}

Se encontraron diferencias estadísticamente significativas, favorables al grupo experimental, en enfoque superficial $F(1,219)=27.966, p<.001$ y en enfoque profundo $F(1,219)=8.76737, p<.01$. También en las puntuaciones parciales de estrategia y motivo, siendo más altas las de enfoque superficial en los sujetos de control y las de profundo en los experimentales.

\subsection{Resultados obtenidos por los sujetos del segundo año separándolos por grupo}

\subsubsection{Análisis intragrupo}

Se presentan los resultados de los análisis intragrupo separando a los alumnos por grupos de clase y profesores.

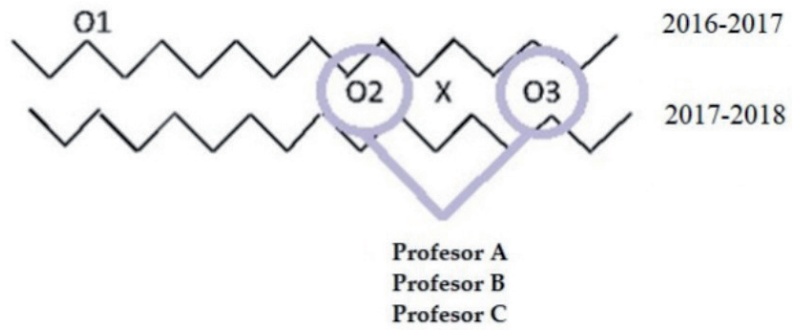

Fuente: Elaboración propia

\subsubsection{Resultados del profesor A}

El profesor A imparte la asignatura en el Grado de Pedagogía.

\subsection{Resultados en estrategias de aprendizaje}

Se encontraron diferencias estadísticamente significativas pretest-postest en la puntuación media global del cuestionario, con mejora en el postest, $p<.01, \eta^{2}$ parcial $=.102$ (tamaño del efecto mediano).

También en la primera escala, de estrategias afectivas, de apoyo y control, $p<$ $.05, \eta^{2}$ parcial $=.063$ (tamaño del efecto pequeño) y en la segunda, de estrategias relacionadas con el procesamiento de la información, $p<.01, \eta^{2}$ parcial $=.123$ (tamaño del efecto mediano).

Así mismo en dos de las seis subescalas: estrategias metacognitivas, $p<.05$, $\eta^{2}$ parcial $=.071 \mathrm{y}$ estrategias de procesamiento y uso, $p<.05, \eta^{2}$ parcial $=.093$ 
(tamaño del efecto mediano). No así en las otras cuatro subescalas, aunque en componentes afectivos se estuvo cerca del nivel de significación, $p=.05$.

Hubo diferencias significativas en cinco estrategias: una de las cuatro metacognitivas: control, autorregulación, $p<.05, \eta^{2}$ parcial $=.067$, y cuatro de las ocho de procesamiento y uso de la información: elaboración, $p<.05, \eta^{2}$ parcial $=.068$, organización, $p<.05, \eta^{2}$ parcial $=.064$, personalización y creatividad, pensamiento crítico, $p<.05, \eta^{2}$ parcial $=.070$, almacenamiento, memorización y uso de los recursos mnemotécnicos, $p<.05, \eta^{2}$ parcial $=.079$.

\subsection{Resultados en enfoques de aprendizaje}

No se encontraron diferencias significativas en ninguna de las dos escalas ni en sus factores, aunque las puntuaciones en enfoque y estrategia profundos estuvieron cercanas al valor de significación de $.05(p=.065$ para el enfoque profundo у $p=.080$ para la estrategia profunda), aumentando las puntuaciones de enfoque profundo y disminuyendo las del superficial.

\subsubsection{Resultados del profesor B}

El profesor B imparte la asignatura en el Grado de Pedagogía.

\subsection{Resultados en estrategias de aprendizaje}

Se encontraron diferencias estadísticamente significativas pretest-postest en la puntuación media global del cuestionario, con mejora en el postest, $p<.05$, $\eta^{2}$ parcial $=.127$. El tamaño del efecto fue mediano.

También en la segunda escala, de estrategias relacionadas con el procesamiento de la información, $p<.01, \eta^{2}$ parcial $=.176$, con un tamaño del efecto grande.

Se dieron diferencias significativas del pretest al postest en tres subescalas: estrategias de búsqueda y selección, $p<.001, \eta^{2}$ parcial $=.218$, y estrategias de procesamiento y uso, $p<.05, \eta^{2}$ parcial $=.116$, con tamaño del efecto grande y estrategias metacognitivas, $p<.05, \eta^{2}$ parcial $=.088$, con tamaño del efecto mediano.

Hubo diferencias significativas en siete estrategias: dos s motivacionales: motivación intrínseca, $p<.05, \eta^{2}$ parcial $=.086$ (tamaño del efecto mediano) y autoeficacia y expectativas, $p<.05, \eta^{2}$ parcial $=.187$ (tamaño del efecto grande); una metacognitiva: conocimiento de objetivos y criterios de evaluación, $p<.01, \eta^{2}$ parcial $=.19$ (tamaño del efecto grande); dos de búsqueda y selección de la información: conocimiento de fuentes y búsqueda de información, $p<.01, \eta^{2}$ parcial $=$ .188 (tamaño del efecto grande), y selección de información, $p<.05$, $\eta^{2}$ parcial $=$ .109 (tamaño del efecto mediano), y dos de procesamiento y uso: personalización y creatividad/pensamiento crítico, $p<.01, \eta^{2}$ parcial $=.162$ (tamaño del efecto grande), y almacenamiento, memorización y uso de recursos mnemotécnicos, $p<$ $.05, \eta^{2}$ parcial $=.089$ (tamaño del efecto mediano). 


\subsection{Resultados en enfoques de aprendizaje}

Se encontraron diferencias estadísticamente significativas en la puntuación global de enfoque profundo $F(1,44)=7.35818, p<.01, \eta^{2}$ parcial $=.14327$, con tamaño del efecto grande y también en las puntuaciones parciales de este enfoque.

Las puntuaciones de enfoque parcial se redujeron en el postest.

\subsubsection{Resultados del profesor C}

El profesor C imparte la asignatura en el Grado de Pedagogía.

\subsection{Resultados en estrategias de aprendizaje}

Se encontraron diferencias estadísticamente significativas pretest-postest en la puntuación media global del cuestionario, con mejora en el postest, $p<.001, \eta^{2}$ parcial $=.262$, con tamaño del efecto grande.

También en la primera escala, de estrategias afectivas, de apoyo y control, $p<$ $.001, \eta^{2}$ parcial $=.263$ y en la segunda, de estrategias relacionadas con el procesamiento, $p<.01, \eta^{2}$ parcial $=.156$, con tamaño del efecto grande.

Así mismo, en cuatro de las seis subescalas: estrategias motivacionales, $p<.05$, $\eta^{2}$ parcial $=.144$, de control del contexto, $p<.001, \eta^{2}$ parcial $=.268$ y de procesamiento y uso, $p<.01, \eta^{2}$ parcial $=.153$, con tamaño del efecto grande. Hubo también diferencias significativas en las estrategias de búsqueda y selección, $p<$ $.05, \eta^{2}$ parcial $=.112$, con tamaño del efecto mediano.

A nivel de estrategias hubo diferencias estadísticamente significativas en nueve: tres estrategias motivacionales: motivación intrínseca, $p<.001, \eta^{2}$ parcial $=.266$, autoeficacia y expectativas, $p<.01, \eta^{2}$ parcial $=.220$, con tamaño del efecto grande, $\mathrm{y}$ atribuciones internas, $F(1,41)=4.437, p<.05, \eta^{2}$ parcial $=.098$, con tamaño del efecto mediano; dos de control del contexto: control del contexto, $p<.001, \eta^{2}$ parcial $=.267$, habilidades de interacción social, $p<.01, \eta^{2}$ parcial $=.187$, siendo en ambas el tamaño del efecto grande; una de las estrategias de búsqueda y selección de la información: selección de la información, $p<.05, \eta^{2}$ parcial $=.102$, con un tamaño del efecto mediano; y tres estrategias de procesamiento y uso: organización, $p<.05$, $\eta^{2}$ parcial $=.148$, con tamaño del efecto grande, almacenamiento/memorización/ uso de recursos mnemotécnicos, $p<.05, \eta^{2}$ parcial $=.102$ y transferencia, uso de la información, $p<.05, \eta^{2}$ parcial $=.103$, con tamaño del efecto mediano.

\subsection{Resultados en enfoques de aprendizaje}

Se encontraron diferencias estadísticamente significativas del pretest al postest en las puntuaciones globales de enfoque superficial, $p<.01, \eta^{2}$ parcial $=.18270$, con tamaño del efecto grande, disminuyendo las puntuaciones en el postest, y también en las puntuaciones parciales. Mejoraron las puntuaciones de enfoque profundo en el postest pero sin diferencias significativas. 
TABLA 3

SíNTESIS DE RESULTADOS

\begin{tabular}{|c|c|c|}
\hline \multicolumn{2}{|c|}{ Análisis de diferencias realizados (ANOVAS) } & Resultados obtenidos \\
\hline $\begin{array}{l}\text { En los sujetos } \\
\text { experimentales } \\
(2016-17)\end{array}$ & $\begin{array}{l}\text { En estrategias de aprendizaje } \\
\text { (pretest-postest) }\end{array}$ & $\begin{array}{l}\text { Diferencias significativas en: Puntuación } \\
\text { global } \\
2 \text { escalas } \\
5 \text { subescalas } \\
16 \text { estrategias }\end{array}$ \\
\hline & $\begin{array}{l}\text { En enfoques de aprendizaje } \\
\text { (pretest-postest) }\end{array}$ & $\begin{array}{l}\text { Diferencias significativas en: } \\
\text { Enfoque superficial (descendió) } \\
\text { Enfoque profundo (se incrementó) }\end{array}$ \\
\hline \multirow{2}{*}{$\begin{array}{l}\text { Entre los sujetos } \\
\text { experimentales } \\
\text { y de control en } \\
\text { postest (2016- } \\
2017 \text { y } 2017- \\
2018)\end{array}$} & En estrategias de aprendizaje & $\begin{array}{l}\text { Diferencias significativas en: } \\
\text { Puntuación global } \\
2 \text { escalas } \\
5 \text { subescalas } \\
18 \text { estrategias }\end{array}$ \\
\hline & En enfoques de aprendizaje & $\begin{array}{l}\text { Diferencias significativas en: } \\
\text { Puntuaciones más altas en enfoque su- } \\
\text { perficial en los sujetos de control y las de } \\
\text { profundo en los experimentales }\end{array}$ \\
\hline \multirow[t]{2}{*}{$\begin{array}{l}\text { Del alumnado } \\
\text { de los grupos } \\
\text { experimentales } \\
\text { del profesor A }\end{array}$} & $\begin{array}{l}\text { En estrategias de aprendizaje } \\
\text { (pretest-postest) }\end{array}$ & $\begin{array}{l}\text { Diferencias significativas en: } \\
\text { Puntuación global } \\
2 \text { escalas } \\
2 \text { subescalas } \\
5 \text { estrategias }\end{array}$ \\
\hline & $\begin{array}{l}\text { En enfoques de aprendizaje } \\
\text { (pretest-postest) }\end{array}$ & $\begin{array}{l}\text { Diferencias significativas en: } \\
\text { Las puntuaciones en el enfoque profundo } \\
\text { y la estrategia profunda estuvieron cerca- } \\
\text { nas al valor de significación }\end{array}$ \\
\hline \multirow[t]{2}{*}{$\begin{array}{l}\text { Del alumnado } \\
\text { de los grupos } \\
\text { experimentales } \\
\text { del profesor B }\end{array}$} & $\begin{array}{l}\text { En estrategias de aprendizaje } \\
\text { (pretest-postest) }\end{array}$ & $\begin{array}{l}\text { Diferencias significativas en: } \\
\text { Puntuación global } \\
1 \text { escala } \\
3 \text { subescalas } \\
7 \text { estrategias } \\
\end{array}$ \\
\hline & $\begin{array}{l}\text { En enfoques de aprendizaje } \\
\text { (pretest-postest) }\end{array}$ & $\begin{array}{l}\text { Diferencias significativas en: } \\
\text { Diferencias estadísticamente significativas en } \\
\text { la puntuación global de enfoque profundo }\end{array}$ \\
\hline \multirow[t]{2}{*}{$\begin{array}{l}\text { Del alumnado } \\
\text { de los grupos } \\
\text { experimentales } \\
\text { del profesor C }\end{array}$} & $\begin{array}{l}\text { En estrategias de aprendizaje } \\
\text { (pretest-postest) }\end{array}$ & $\begin{array}{l}\text { Diferencias significativas en: } \\
\text { Puntuación global } \\
2 \text { escalas } \\
4 \text { subescalas } \\
9 \text { estrategias }\end{array}$ \\
\hline & $\begin{array}{l}\text { En enfoques de aprendizaje } \\
\text { (pretest-postest) }\end{array}$ & $\begin{array}{l}\text { Diferencias significativas en: } \\
\text { Incremento de puntuaciones globales de } \\
\text { enfoque superficial, disminuyendo las } \\
\text { puntuaciones en el postest }\end{array}$ \\
\hline
\end{tabular}

Fuente: Elaboración propia 


\section{Discusión Y CONCLUSIONES}

El primer objetivo de este trabajo era evaluar el impacto de la aplicación de un formato metodológico centrado en el aprendizaje en el modo de aprender de estudiantes universitarios, concretado en sus estrategias y enfoques de aprendizaje.

Analizando la evolución del pretest al postest (análisis intragrupo) de los sujetos del grupo experimental conjunto los resultados fueron muy positivos: se produjo una mejora notable en las puntuaciones de estrategias de aprendizaje del alumnado. Mejoró significativamente la puntuación global del cuestionario y también las de las dos escalas y las de cinco de las seis subescalas (motivacionales, metacognitivas, de control del contexto, de búsqueda y selección y de procesamiento). Así mismo, se produjeron mejoras estadísticamente significativas en dieciséis de las veinticinco estrategias evaluadas.

Hubo diferencias estadísticamente significativas pretest-postest en las puntuaciones globales de enfoque profundo y superficial y en las parciales, de motivo y estrategia, incrementándose el enfoque profundo y disminuyendo el superficial en el postest.

También se compararon las puntuaciones obtenidas en el postest por los sujetos del Grupo 1 y los del Grupo 2 (análisis intergrupos). El Grupo 1 actuaba como grupo de control, al haber utilizado sus profesores metodología de tipo tradicional. El Grupo 2 había recibido intervención específica centrada en el aprendizaje durante el curso 2017-18.

Se dieron diferencias estadísticamente significativas, a favor de los sujetos experimentales, en la puntuación global del cuestionario, en las de las dos escalas, en cinco de las seis subescalas y en dieciocho de las veinticinco estrategias.

En enfoques de aprendizaje hubo diferencias estadísticamente significativas, a favor de los sujetos del grupo experimental, con puntuaciones más altas en enfoque profundo y más bajas en enfoque superficial.

Los resultados muestran los efectos positivos del uso de metodologías centradas en el aprendizaje, sobre el modo de aprender del alumnado universitario, que mejora sus estrategias y sus enfoques de aprendizaje. Los resultados son claros cuando se analiza la evolución del pretest al postest de los sujetos del Grupo 2 y también cuando se comparan en el postest los resultados de los dos grupos, el Grupo 1 (sujetos del curso 2016-17, que cursaron la misma asignatura con dos de los profesores, que utilizaban metodología tradicional) y el Grupo 2 (sujetos del curso 2017-18, que cursaron la misma materia con los dos profesores anteriores y otro profesor, haciendo uso de métodos centrados en el aprendizaje). La comparación intergrupos da más solidez a los resultados al hacer uso de un diseño cuasiexperimental.

El segundo objetivo era analizar la influencia del profesor en la eficacia del formato metodológico centrado en el aprendizaje. En este caso se separaron los alumnos del segundo año (Grupo 2) por grupos de clase y profesores (A, B y C).

Los tres grupos mejoraron. 
El grupo de alumnos del profesor A lo hizo en estrategias - puntuación global, las de las dos escalas, dos de las seis subescalas y cinco de las veinticinco estrategias-, pero no en enfoques.

El grupo del profesor B obtuvo mejores puntuaciones en estrategias - puntuación global, primera escala, tres de las seis subescalas y siete estrategias- y en enfoques (incrementándose las puntuaciones de enfoque profundo).

Y el del profesor C lo hizo en estrategias — puntuación global, las dos escalas, cuatro de las seis subescalas y nueve estrategias-y enfoques — disminuyeron las puntuaciones de enfoque superficial-.

Los resultados fueron ligeramente mejores en el grupo del profesor C, seguidos por el B y por el A. Así pues, aunque los métodos hayan sido los mismos, la variable profesor supone un elemento modulador de los resultados de la intervención, tal como se suponía.

Nos parece que la mejora tiene una clara lógica, habida cuenta de los métodos utilizados. Para funcionar bien con estos métodos, más participativos y potenciadores de la autonomía del estudiante, éste necesita de más compromiso, más implicación, una buena planificación y también capacidad de autoevaluación y autorregulación, así como de un enfoque profundo de aprendizaje. El alumnado debe mantener una actitud activa con la materia, realizando trabajo autónomo antes de las clases, con indagación personal a partir de las cuestiones que debe responder, y también durante la clase, dado que las cuestiones se debaten. También ha de desarrollar habilidades de trabajo cooperativo y de autorregulación para llevar adelante con solvencia el trabajo de grupo. La exigencia de elaboración de un portafolios enfatiza, así mismo, las habilidades y actitudes que estamos comentando, específicamente las habilidades metacognitivas, trabajadas en dicho portafolios que incluye preguntas de reflexión sobre el proceso seguido para resolver la tarea.

En la literatura hay ejemplos similares al nuestro, con muestras no demasiado grandes, en unos casos con resultados positivos y en otros no tanto:

Así, el caso del estudio de Li y Guo (2015), que introdujeron métodos centrados en el aprendizaje, con una muestra universitaria pequeña, utilizando trabajo cooperativo, con una valoración positiva del alumnado.

También Chen Zhou et al. (2015) rediseñaron un curso de laboratorio de fisiopatología, para potenciar el pensamiento crítico, la autogestión del aprendizaje mediante un aprendizaje más activo. Con un diseño cuasiexperimental los resultados del grupo experimental fueron significativamente mejores tanto en pruebas de laboratorio como en la valoración del alumnado frente al de control, que usaba métodos tradicionales.

García-Carpintero (2017) introdujo el portafolios como método de enseñanza, aprendizaje y evaluación en un grupo de alumnos de $4^{\circ}$ curso de Enfermería de una universidad española y las valoraciones del alumnado fueron positivas.

Turner y Webster (2017) introdujeron el método de clase invertida en un curso de ingeniería usando discusiones en grupo y solución de problemas. Los autores compararon sus resultados con los de otros alumnos que seguía metodología 
Y LOS ENFOQUES DE APRENDIZAJE DE LOS UNIVERSITARIOS

tradicional y encontraron resultados ligeramente mejores en resolución de problemas y en contenido técnico, pero también una reacción negativa frente a métodos instruccionales, no familiares para ellos.

Maya Díaz et al. (2021) utilizaron el método de clase invertida para potenciar la implicación y el compromiso del alumnado de tres asignaturas del Grado de Química de la Universidad de Sevilla y una del Grado de Química de la Universidad de Barcelona. Usaron un diseño cuasiexperimental con grupo de control de metodología tradicional (105 sujetos) y con grupo experimental que utilizaba clase invertida (147), obteniéndose mejora de resultados académicos en pruebas sobre los contenidos trabajados.

Medina et al. (2021) usaron una combinación de clase invertida y aprendizaje en equipo en cinco grupos con 318 alumnos de $2^{\circ}$ curso del grado de ADE de la Universidad de Barcelona, logrando un impacto positivo en los resultados obtenidos en cuestionarios de los contenidos impartidos.

Si bien es cierto que la mayoría de estos estudios refieren resultados positivos, también lo es que esos resultados derivan de valoraciones del alumnado o del uso de cuestionarios de contenidos puntuales. En nuestro caso, los resultados revelaron mejoras significativas en las estrategias y enfoques de aprendizaje del alumnado, evaluando estos dos constructos mediante cuestionarios validados y muy utilizados en la literatura científica (CPE y CEVEAPEU). Estamos convencidos, por los datos obtenidos, que esos resultados derivan del cambio producido en el modelo de formación utilizado, centrado en el aprendizaje, que comportó una reorientación general de los procesos de enseñanza-aprendizaje enseñanza y evaluación (Arroyo, 2018), teniendo como leitmotiv el aprendizaje autónomo del alumnado, la construcción conjunta del conocimiento y la potenciación de su autorregulación. Eso explica, desde nuestra perspectiva, el impacto de nuestro planteamiento en los procesos de aprendizaje del alumnado, estrategias y enfoques. No nos quedamos, como en la mayoría de los estudios, en una sola técnica específica. Desde nuestro punto de vista, esa es una limitación de los estudios analizados, que se quedaron en iniciativas puntuales, circunscritas a cambios muy reducidos.

Los resultados obtenidos son coherentes con los hallados en otros trabajos previos que muestran el impacto del uso de métodos centrados en el aprendizaje en las capacidades del alumno y en su percepción del entorno de aprendizaje (Gargallo, Cebriá, et al., 2017; Gargallo et al., 2018), en los estilos (Gargallo, Pérez, et al., 2017) y en las estrategias y el rendimiento (Gargallo et al., 2014).

Como conclusión, querríamos manifestar que lo ideal para que el enfoque centrado en el aprendizaje cobrara su verdadera dimensión y se generalizara habría implementar estos métodos en titulaciones y en centros completos, incluso en toda la universidad, si fuera posible. Éste es el caso de la investigación de Kember (2009), que articula un modelo de trabajo para llevar a cabo la integración de la enseñanza centrada en el aprendizaje en una universidad de Hong-Kong.

Para ello se utilizaron entrevistas con profesores especialmente buenos para delimitar protocolos y modelos de buenas prácticas, formación para profesorado 
joven, puesta en marcha de proyectos innovadores implementados por profesorado comprometido con este modelo, evaluación de los proyectos innovadores y revisión de la calidad de estos proyectos por el profesorado con ayuda de un experto externo.

Kember (2009) aporta datos de éxito parcial de la implementación, en la percepción de los alumnos del entorno de aprendizaje orquestado por los profesores y en el desarrollo de capacidades del alumnado. El autor prevé completar los datos una vez concluido el proceso, en futuras publicaciones, que no hemos conseguido encontrar.

Otra iniciativa interesante es la de Lavoie y Rosman (2007), en la Universidad de Connecticut. Para llevarla a cabo plantean un programa de formación de profesores, usando un enfoque centrado en el estudiante para que los profesores - estudiantes ahora - lo puedan llevar a cabo posteriormente con su alumnado aprendiendo a diseñar sus cursos con este enfoque. Los autores refieren mejoras en el aprendizaje y un compromiso más activo del alumnado, que percibe mayor coherencia en el planteamiento.

No es sencillo pasar de un enfoque tradicional a otro centrado en el aprendizaje (Heise y Himes, 2010), cuando se es ambicioso y se quiere implementar en titulaciones, centros y en la universidad en su conjunto, y puede haber resistencias duras (Barr y Tagg, 1995), porque toda la estructura universitaria puede verse afectada. Un proceso de esta índole exige cambios en la filosofía de la organización, en la organización misma, y políticas institucionales pertinentes (De La Sablonnière et al., 2009). Es necesario romper las rígidas fronteras de los departamentos y de las áreas de conocimiento lograr la implicación del profesorado (Tracy y Gutiérrez, 2019). Para ello es imprescindible su formación de cara a lograr su compromiso. Los profesores más veteranos son menos proclives que los jóvenes a cambiar (Blumberg, 2016). Y también se precisa la implicación de los estudiantes (Maclellan, 2008).

Mientras no se consoliden iniciativas institucionales como las reseñadas, los resultados que presentamos pueden servir para animar al profesorado a utilizar métodos centrados en el aprendizaje, habida cuenta de las consecuencias positivas de su utilización y de que es algo relativamente fácil de implementar.

No queremos terminar sin hacer mención a algunas limitaciones del estudio. La la muestra no es representativa de la población ni de la universidad. Es lo más habitual en este tipo de estudios por la dificultad de conseguir muestras representativas y de trabajar con ellas en los procesos de formación. Por otra parte, los diseños cuasiexperimentales son los suficientemente robustos, al garantizar la existencia de grupos de control. Otra limitación es que hay un cierto desequilibrio entre los grupos experimental y de control derivado de que en el primer curso sólo se pudieron utilizar dos de los grupos, por la no participación del profesor del tercer grupo, y también por un menor $\mathrm{N}$ en los dos grupos del primer curso frente a los del segundo.

A pesar de estas limitaciones, creemos que nuestro estudio aporta datos relevantes y puede servir a los investigadores y al profesorado para avanzar. 
LAURA BARBOYON COMBEY Y BERNARDO GARGALLO LÓPEZ

MÉTODOS CENTRADOS EN EL ESTUDIANTE. SUS EFECTOS EN LAS ESTRATEGIAS

Y LOS ENFOQUES DE APRENDIZAJE DE LOS UNIVERSITARIOS

\section{REFERENCIAS BIBLIOGRÁFICAS}

Arman, M. S. (2018). Student-centered approach to teaching: It takes two to tango. The Ahfad Journal, 35(2), 64-71.

Arroyo, M. A. (2018). A learning-centered approach to remote learning. UNLBest Teaching Practices Expo. 147. https://bit.ly/2NjTuab

Ato, M., y Vallejo, G. (2007). Diseños experimentales en Psicología. Ediciones Pirámide.

Barr, R. B. \& Tagg, J. (1995). From Teaching to Learning. A New Paradigm for Undergraduate Education. Change, 27(6), 13-25. https://doi.org/10.1080/00091383.1995.10544672

Biggs, J. (2005). Calidad del aprendizaje universitario. Narcea.

Biggs, J., Kember, D., \& Leung, D. Y. P. (2001). The revised two-factor Study Process Questionnaire: R-SPQ-2. British Journal of Educational Psychology, 71, 133-149. https://doi. org/10.1348/000709901158433

Blumberg, Ph. (2016). Factors that influence faculty adoption of learning-centered approaches. Innovative Higher Education, 41, 303-315. https://doi.org/10.1007/s10755-015-9346-3

Campbell, D. T., \& Stanley, J. C. (1979). Diseños experimentales y cuasiexperimentales en la investigación social. Amorrortu.

Carwile, J. (2007). A Constructivist Approach to Online Teaching and Learning. Inquiry, 12(1), 68-73. https://files.eric.ed.gov/fulltext/EJ833907.pdf

CE. (2007). Comunicado de Londres https://bit.ly/3tSbTe4

CE. (2009). Comunicado de Lovaina. https://bit.ly/3aFAXNL

CE. (2012). Comunicado de Bucarest. https://bit.ly/3sRaF1p

CE. (2015). Comunicado de Yerevan. https://bit.ly/3xs6vR2

Chen, J., Zhou, J., Sun, L., Wu, Q., Lu, H., \& Tian, J. (2015). A new approach for laboratory exercise of pathophysiology in China based on student-centered learning. Advances in Physiology Education, 39, 116-119. https://doi.org/10.1152/advan.00143.2014

De La Sablonnière, R., Taylor, D. M., \& Sadykova, N. (2009). Challenges of applying a student-centred approach to learning in the context of Education in Kyrgyzstan. International Journal of Educational Development, 29, 628-634. https://doi.org/10.1016/j. ijedudev.2009.01.001

EI-ESU. (2010). Student-Centred Learning. An Insight into Theory and Practice. Education International, European Students' Union.

Emaliana, I. (2017). Teacher-centered or student-centered learning approach to promote learning? Journal Social Humaniora, 10 (2), 59-70. https://oaji.net/articles/2017/5501-1519102561. pdf

Entwistle, N., \& Peterson, E. (2004). Learning styles and approaches to studying. In C. Spielberger (Ed.), Encyclopedia of Applied Psycology, 2 (pp. 537-542). Elsevier.

García-Carpintero, E. (2017). El portafolios como metodología de enseñanza-aprendizaje y evaluación en el prácticum: percepciones de los estudiantes. REDU/Revista de Docencia Universitaria, 15(1), 241-257. https://doi.org/10.4995/redu.2017.6043

García-Valcárcel, A. (1993). Análisis de los modelos de enseñanza empleados en el ámbito universitario, Revista Española de Pedagogía, 194, 27-53. https://bit.ly/3vsHRyC

Gargallo, B. (Coord.). (2017). Enseñanza centrada en el aprendizaje y diseño por competencias en la universidad. Fundamentación, procedimientos y evidencias de aplicación e investigación. Tirant lo Blanch. 
LAURA BARBOYON COMBEY Y BERNARDO GARGALLO LÓPEZ MÉTODOS CENTRADOS EN EL ESTUDIANTE. SUS EFECTOS EN LAS ESTRATEGIAS Y LOS ENFOQUES DE APRENDIZAJE DE LOS UNIVERSITARIOS

Gargallo, B., Cebriá, M. A., Sarriá, B., Benavent, A., y Queralt, A. (2017). Métodos centrados en el aprendizaje en Fisioterapia. Capacidades del alumno y contexto de aprendizaje. REDU. Revista de docencia universitaria, 15 (2), 329-347. https://doi.org/10.4995/redu.2017.7868

Gargallo, B., Garfella, P. R., Sahuquillo, P., Verde, I., y Jiménez, M. A. (2015). Métodos centrados en el aprendizaje, estrategias y enfoques de aprendizaje en estudiantes universitarios. Revista de Educación, 370, 229-254. https://doi.org/10.4438/1988-592X-RE-2015-370-304

Gargallo, B., Morera, I., y García, E. (2015). Metodología innovadora en la universidad. Sus efectos sobre los procesos de aprendizaje de los estudiantes universitarios. Anales de psicología, 31(3), 901-915. https://doi.org/10.6018/analesps.32.1.179871

Gargallo, B., Morera, I., Iborra, S., Climent, M. J., Navalón, S., y García, E. (2014). Metodología centrada en el aprendizaje. Su impacto en las estrategias de aprendizaje y el rendimiento de los estudiantes universitarios. Revista Española de Pedagogía, 259, 415-435. https:// bit.ly/3volL0j

Gargallo, B., Sahuquillo Mateo, P. M., Verde, I., Almerich, G. (2018). ¿Qué ocurre cuando los profesores utilizan métodos centrados en el aprendizaje? Efectos en los enfoques de aprendizaje, en las capacidades del alumno y en su percepción del entorno de aprendizaje. Revista de Educación, 382, 163-190. https://doi.org/10.4438/1988-592X-RE-2018-382-396

Gargallo, B., Suárez, J. M., y Pérez, C. (2009). El cuestionario CEVEAPEU. Un instrumento para la evaluación de las estrategias de aprendizaje de los estudiantes universitarios, RELIEVE, 15(2), 1-31. https://www.uv.es/RELIEVE/v15n2/RELIEVEv15n2_5.htm

Gargallo, B., Pérez, C., Verde, I., y García, E. (2017). Estilos de aprendizaje en estudiantes universitarios y enseñanza centrada en el aprendizaje. RELIEVE, 23(2), art. 2. https:// doi.org/10.7203/relieve.23.2.9078

Gow, L., \& Kember, D. (1993). Conceptions of teaching and their relationship to student learning. British Journal of Educational Psychology, 63, 20-33. https://doi. org/10.1111/j.2044-8279.1993.tb01039.x

Heise, B. A., \& Himes, D. (2010). Educational Innovation: The Course Council-An example of student-centered learning. Journal of Nursing Education, 49(6), 343-345. https://doi. org/10.3928/01484834-20100115-04

Jiménez Hernández, D., González Ortiz, J. J., y Tornel Abellán, M. (2020). Metodologías activas en la universidad y su relación con los enfoques de enseñanza. Profesorado. Revista de currículum y formación del profesorado, 24 (1), 76-94. https://doi.org/10.30827/ profesorado.v24i1.8173

Kember, D. (2009). Promoting student-centred forms of learning across an entire university. Higher Education, 58, 1-13. https://doi.org/10.1007/s10734-008-9177-6

Kember, D., \& Gow, L. (1994). Orientations to teaching and their effects on the quality of student learning. Journal of Higher Education, 65(1), 59-74. https://doi.org/10.2307/2943877

Kember, D., \& Kwan, K. (2000). Lecturers' approaches to teaching and their relationship to conceptions of good teaching. Instructional Science, 28, 469-490. https://link.springer. com/content/pdf/10.1023/A:1026569608656.pdf

Lavoie, D., \& Rosman, A. J. (2007) Using active student-centred learning-based instructional design to develop faculty and improve course design, delivery, and evaluation. Issues in Accounting Education, 22(1), 105-118. https://shorturl.at/gpqH5

Li, L. \& Guo, R. (2015). A student-centered guest lecturing: A constructivism approach to promote student engagement. Journal of Instructional Pedagogies, 15, 1-7. https://files. eric.ed.gov/fulltext/EJ1060070.pdf 
LAURA BARBOYON COMBEY Y BERNARDO GARGALLO LÓPEZ

MÉTODOS CENTRADOS EN EL ESTUDIANTE. SUS EFECTOS EN LAS ESTRATEGIAS

Y LOS ENFOQUES DE APRENDIZAJE DE LOS UNIVERSITARIOS

Maclellan, E. (2008). The significance of motivation in student-centred learning: a reflective case-study. Teaching in Higher Education, 13(4), 411-421. https://doi. org/10.1080/13562510802169681

Maya Díaz, C., Iglesias Sigüenza, J. y Giménez, X. (2021). Clase Invertida Síncrona en asignaturas STEM. Revista de Educación, 391, 15-41. https://doi.org/10.4438/1988592X-RE-2021-391-469

McCoy, L., Pettit, R. K., Kellar, Ch., \& Morgan, (2018). Tracking active learning in the medical school curriculum: a learning-centered approach. Journal of Medical Education and Curriculum Development, 5, 1-9. https://doi.org/10.1177/2382120518765135

McCune, V., \& Entwistle, N. (2011). Cultivating the disposition to understand in $21^{\text {st }}$ century university education. Learning and Individual Differences, 21(3), 303-310. https://doi. org/10.1016/j.lindif.2010.11.017

Medina, J.L., Hervas, G., y Cairó-i-Céspedes, G. (2021). Aprendizaje del análisis económico de la desigualdad a través del «Team-based learning». Revista de Educación, 391, 43-64. https://doi.org/10.4438/1988-592X-RE-2021-391-470

Motjolopane, I. (2021). Teaching research methodology: student-centerd approach computina education undergraduate course. Emerging Science Journal, 5(1), 34-43. https:// ijournalse.org/index.php/ESJ/article/view/365

Pintrich, P. R. (2000). The role of goal orientation in self-regulated learning. In M. Boekaerts, P. Pintrich \& M. Zeidner (Eds.), Handbook of Self-Regulation (pp. 451-502). Academic Press.

Pintrich, P. R. (2004). A conceptual framework for assessing motivation and self-regulated learning in college students. Educational Psychology Review, 16(4), 385-407. https:// link.springer.com/content/pdf/10.1007/s10648-004-0006-x.pdf

Ruiz Lara, E., Hernández Pina. F. y Ureña Villanueva, F. (2008). Enfoques de aprendizaje y rendimiento institucional y afectivo de los alumnos de la titulación de Ciencias de la Actividad Física y el Deporte. Revista de Investigación Educativa, 26(2), 307-322. https:// revistas.um.es/rie/article/view/93941/90581

Samuelowicz, K. \& Bain, J. D. (1992). Conceptions of teaching held by academic teachers. Higher Education, 22, 229-249. https://doi.org/10.1007/BF00138620

Samuelowicz, K., \& Bain, J. D. (2001). Revisiting academics' beliefs about teaching and learning. Higher Education, 41, 299-325. https://doi.org/10.1023/A:1004130031247

Serin, H. (2018). A comparison of teacher-centered and student-centered approaches in educational settings. International Journal of Social Sciences E Educational Studies, 5(1), 164-167. https://doi.org/10.23918/ijsses.v5i1p164

Shadish, W. R., Cook, T. D., \& Campbell, D. T. (2002). Experimental and quasi-experimental designs for generalized causal inference. Houghton Mifflin Company.

Tracy, P. J., \& Gutiérrez, A. R. (2019). A situated, learning-centered approach to designing a new faculty program. The Journal of Faculty Development, 33(3), 95-102. https://bit. ly/3gGOWHc

Turner, M. J. \& Webster, R. D. (2017). A comparison of delivery formats to encourage studentcentered learning in a power engineering technology course. American Journal of Engineering Education, 8(7), 141-156. https://doi.org/10.19030/ajee.v8i2.10071

Yip, M. C. W. (2012). Learning strategies and self-efficacy as predictors of academic performance: a preliminary study. Quality in Higher Education, 18(1), 23-34. https://doi.org/ $10.1080 / 13538322.2012 .667263$

Zimmerman, B. J. (2002). Becoming a self-regulated learner: an overview. Theory into Practice, 41, 64-70. https://doi.org/10.1207/s15430421tip4102_2 